Published in final edited form as:

Stat Methods Med Res. 2018 July ; 27(7): 2038-2049. doi:10.1177/0962280216673675.

\title{
A Bayesian hierarchical model for demand curve analysis
}

\author{
Yen-Yi Ho\#1, Tien Nhu Vo\#2, Haitao $\mathrm{Chu}^{3}$, Xianghua Luo ${ }^{3,4}$, and Chap T Le ${ }^{3,4}$ \\ ${ }^{1}$ Department of Statistics, College of Arts and Sciences, University of South Carolina, South \\ Carolina, SC, USA \\ 2Division of Epidemiology, School of Public Health, University of Minnesota, Minnesota, MN, USA \\ ${ }^{3}$ Division of Biostatistics, School of Public Health, University of Minnesota, Minnesota, MN, USA \\ ${ }^{4}$ Masonic Cancer Center, University of Minnesota, Minnesota, MN, USA \\ \# These authors contributed equally to this work.
}

\begin{abstract}
Drug self-administration experiments are a frequently used approach to assessing the abuse liability and reinforcing property of a compound. It has been used to assess the abuse liabilities of various substances such as psychomotor stimulants and hallucinogens, food, nicotine, and alcohol. The demand curve generated from a self-administration study describes how demand of a drug or non-drug reinforcer varies as a function of price. With the approval of the 2009 Family Smoking Prevention and Tobacco Control Act, demand curve analysis provides crucial evidence to inform the US Food and Drug Administration's policy on tobacco regulation, because it produces several important quantitative measurements to assess the reinforcing strength of nicotine. The conventional approach popularly used to analyze the demand curve data is individual-specific nonlinear least square regression. The non-linear least square approach sets out to minimize the residual sum of squares for each subject in the dataset; however, this one-subject-at-a-time approach does not allow for the estimation of between- and within-subject variability in a unified model framework. In this paper, we review the existing approaches to analyze the demand curve data, non-linear least square regression, and the mixed effects regression and propose a new Bayesian hierarchical model. We conduct simulation analyses to compare the performance of these three approaches and illustrate the proposed approaches in a case study of nicotine selfadministration in rats. We present simulation results and discuss the benefits of using the proposed approaches.
\end{abstract}

\section{Keywords}

Bayesian hierarchical model; mixed effects regression; non-linear least square regression; demand curve analysis; prism

\footnotetext{
Reprints and permissions: sagepub.co.uk/journalsPermissions.nav

Corresponding author: Yen-Yi Ho, Department of Statistics, University of South Carolina 216 LeConte College, University of South Carolina, 1523 Greene Street, Columbia, SC 29208, USA. Hoyen@stat.sc.edu.

Declaration of conflicting interests

The author(s) declared no potential conflicts of interest with respect to the research, authorship, and/or publication of this article.
} 


\section{Introduction}

Relative reinforcing efficacy (RRE) is a fundamental concept frequently used in substance abuse research. ${ }^{1,2}$ It describes the reinforcing potency and abuse liability of a substance. A self-administration experiment is a valuable tool to assess RRE. Self-administration studies have been widely employed to study the reinforcing strength of various substances such as psychomotor stimulants and hallucinogens, ${ }^{1,3}$ food,4 nicotine, ${ }^{5,6}$ and alcohol.7 The demand curve data obtained from a self-administration experiment describe how demand of a drug or non-drug reinforcer varies as a function of price. 8 According to the law of demand, the consumption of a commodity generally decreases when its price increases; as such, conceptually, the demand curve is always downward sloping.

The approval of the 2009 Family Smoking Prevention and Tobacco Control Act incentivizes research to determine the RRE of nicotine in order to inform the US Food and Drug Administration (FDA) about individual characteristics (such as gender, smoking history) in response to nicotine-reduction tobacco products, the feasibility of a nicotine-reduction policy, and its potential public health impact. Animal self-administration studies provide vital evidence to support the FDA's future policy on tobacco product regulation because rodent models of nicotine self-administration (NSA) are the gold standard for assessing reinforcing properties of nicotine.6 In addition, human self-administration studies, such as cigarette purchase tasks, suffer from issues such as reporting errors, non-compliance, and loss of follow-up.

A number of models have been proposed to perform demand curve analysis; ${ }^{9,10}$ the most recent and popularly used model is the exponential demand curve, which has the following form

$$
\log Q=\log Q_{0}+K\left(e^{-\alpha P}-1\right)
$$

In equation (1), $Q$ is the quantity consumed, $Q_{0}$ is the amount consumed when the unit price $P$ of a good approaches 0 . For example, in animal and human research on drug addiction, $Q_{0}$ is the level of consumption of nicotine, cigarettes, or other abused drugs, when there is no cost or effort required to obtain those commodities. Other parameters in the demand curve described in equation (1) are $K$ and $a$ (a measure of demand curve elasticity). Elasticity describes the rate of decline in relative log consumption, i.e. how quickly consumption falls with increases in price. According to Hursh and Silberberg, $9 a$ is the measure of reinforcing strength.

Another two clinically relevant quantities related to the demand curve are $O_{\max }$ and $P_{\max }$. $O_{\max }$ is the maximum expense (price times consumption) a person is willing to spend for a commodity; $P_{\max }$ is the corresponding unit price. $O_{\max }$ is clinically important because of its correlation with clinical addiction indices in both alcohol and nicotine studies.11 It can be calculated by the following equation 
$$
O_{\text {max }}=P_{\text {max }} Q=P_{\max } Q_{0} e^{K\left(e^{-\alpha P_{\max }}-1\right)}
$$ \\ $P_{\max }$ does not have a closed form solution and is obtained by solving the following equation

$$
K \alpha P_{\max } e^{-\alpha P \max }=1
$$

The conventional method popularly used to estimate exponential demand curve is the individual-specific nonlinear least squares regression model (NLIN), $9,7,11$ which can be implemented through GraphPad Prism software. The NLIN method uses a three-step approach. First the parameter $K$ in equation (1) is determined based the range of the data. $K$ is calculated as the difference of maximum and minimum observed consumptions in the data in $\log _{e}$ unit and set as a constant across all animals in the data. Second, the individual values of $a$ and $Q_{0}$ are determined by minimizing the residual sum of squares, one-subject-at-atime. Finally, the overall $a, Q_{0}, P_{\max }$ and $O_{\max }$ estimates are calculated as the mean of individual $a, Q_{0}, P_{\max }$, and $O_{\max }$ estimates obtained from each subject. The $95 \%$ confidence intervals for $a, Q_{0}, P_{\max }$ and $O_{\max }$ estimates are obtained by assuming the estimates are normally distributed.

There are several concerns in using the conventional approach for exponential demand curve analysis: (1) The estimation of $P_{\max }$ and $O_{\max }$ estimates rely heavily on graphical methods. 8,11 Although graphical methods could be straightforward, they have limitations in that the resolution of estimating $P_{\max }$ and $O_{\max }$ is restricted by the sample rate of price.11 (2) The ad hoc estimation of $K$ might have large impacts on the estimations of other parameters. (3) Current approaches do not obtain the standard errors for $P_{\max }$ and $O_{\max }$ in a model-based framework. These issues are addressed using alternative approaches considered in this study: mixed effects regression and a Bayesian hierarchical model.

A left-censored mixed effects regression method has been considered by Liao et al.12 to model the exponential demand curve in settings with zero consumption. In order to obtain the standard deviation of $P_{\max }$ and $O_{\max }$ in the mixed effects model framework, we proposed to use the bias-corrected and accelerated bootstrap approach13 due to the skewness of $P_{\max }$ and $O_{\max }$ distribution. On the other hand, the Bayesian approach proposed in this study offers a straightforward approach to compute means, standard deviations, and 100(1-a) percent equal-tail credible intervals (equal-tail CIs) for $P_{\max }$ and $O_{\max }$ based on posterior distributions. This is particularly crucial because the distribution of $P_{\max }$ and $O_{\max }$ is often skewed. As a result of major developments of computational algorithms, 14 the use of Bayesian approaches is rapidly increasing. 15

In this paper, we discuss statistical inference and compare the performance of the three approaches: the NLIN, mixed effects approach via maximum likelihood (ML) estimation method, and the proposed Bayesian approach via the Markov chain Monte Carlo (MCMC) method, with a focus on small-to-moderate sample sizes. 
In Section 2, we describe a motivating case study that estimated demand curves in rats selfadministering nicotine during progressive unit-dose reduction.5 In Section 3, we describe the mixed effects regression and Bayesian hierarchical model. In Section 4, we present the implementation of the Bayesian hierarchical model using data from the case study. The simulation studies to compare the performance of the three approaches are presented in Section 5. Finally, a discussion of various approaches is presented in Section 6.

\section{Material and study design}

The data were collected from a study designed to model a nicotine-reduction policy in rats by arranging progressive decreases in the unit dose of nicotine to the point of extinction of self-administration in every subject. The study aimed to examine the gender difference in NSA in rats. 5 Seven male and seven female Holtzman rats were trained to self-administer nicotine during daily 23 -h sessions. The price of nicotine - defined as the number of responses required per unit dose16-was then increased weekly by reducing the unit dose of nicotine until responding fell to within the range of extinction levels. The amount of nicotine consumed $(\mathrm{mg} / \mathrm{kg} / \mathrm{day})$ by each rat was recorded at each unit price. More details about the experimental procedures are described in Grebenstein et al.5 The data are represented in Table 1 , and each value is the mean of three 23 -h sessions. The blank cells in Table 1 indicate that the consumption of nicotine fell to within the range of extinction levels.

\section{Statistical methods}

\subsection{Mixed effects model}

Let $y_{i j}$ be the log consumption of nicotine for the ith rat at the $j$ th price $P_{j}$. In the data under the study, the model can be written as follows

$$
y_{i j}=\left[C_{0}+C_{M} I\left(\text { Male }_{i}\right)+u_{i}\right]+e^{\log K}\left\{e^{-\left[\left(\alpha_{0}+\alpha_{M} I\left(\text { Male }_{i}\right)+b_{i}\right) P_{j}\right]}-1\right\}+\varepsilon_{i j}
$$

where $C_{M}$ is the difference in the log consumption between male and female rats when the price $P_{f}=0 ; a_{0}$ represents the rate of decline; $a_{M}$ captures the group difference of the rate of decline between male and female rats. The two random effects $u_{i}$ and $b_{i}$ represent the log consumption at price 0 and the rate of decline for each rat, respectively. These random effects follow a bivariate normal distribution with mean 0 and a covariance matrix structure $\sum=\left[\begin{array}{cc}\sigma_{u}^{2} & \rho \sigma_{u} \sigma_{b} \\ \rho \sigma_{u} \sigma_{b} & \sigma_{b}^{2}\end{array}\right]$, and $\rho$ represents the correlation between the log consumption at price 0 and reinforcing value. The correlation of the log consumption of nicotine $y_{i j}$ across different $j$ for a given rat $i$ is modeled through the random effects. Given the random effects $u_{i}$ and $b_{i}$, the error terms $\varepsilon_{i j}$ for a given rat $i$ are independent and were assumed to follow a normal distribution with mean 0 and variance $\sigma^{2}$. In this model framework for male rats, the log consumption at price zero is $C_{0}+C_{M}$, and the rate of decline in consumption is $a_{0}+a_{M}$. 
Table 2 shows the analysis results using NLIN and mixed effects model. The results using the NLIN approach have been presented and described in Grebenstein et al.5 The results using two approaches yield consistent conclusion; however, the 95\% CI estimates using the three-step NLIN approach assume that the parameter estimates follow normal distribution. This assumption can be violated when sample size is small or when the distributions of the estimators are skewed. In addition, the results in Table 2 also suggested that the ad hoc estimation of $K$ has considerable impact on the estimations of other parameters using the NLIN approach.

In the mixed effects model, when the price is close to 0 , indicating that there is no effort needed, female rats consume $e^{0.906}=2.48 \mathrm{mg} / \mathrm{kg}$ of nicotine: this is statistically significant with $95 \%$ CI of $(1.93,3.18)$. Male rats initially consumed less nicotine in log scale than female rats; however, this is not statistically significant, because the parameter estimate for $C_{M}$ is -0.25 with $95 \% \mathrm{CI}$ of $(-0.60,0.10)$. The reinforcing strength for female rats $\left(a_{0}\right)$ is $1.053 \times 10^{-3}$ and is statistically significantly different from zero, with a $95 \% \mathrm{CI}$ of $(6.58 \times$ $\left.10^{-4}, 1.45 \times 10^{-3}\right)$. Male rats have a lower rate of decline in nicotine consumption than female rats; however, this is not statistically significant because the parameter estimate $a_{M}$ is $-1.93 \times 10^{-4}$, with a $95 \% \mathrm{CI}$ of $\left(-7.34 \times 10^{-4}, 3.48 \times 10^{-4}\right)$. The result suggests that there was no sex difference in the reinforcing efficacy of nicotine. The overall $R^{2}$ using the mixed effects model is 0.9865 .

After obtaining the estimates of $K, a_{0}, a_{M}$, we calculated $P_{\max }$ by solving equation (3) and then calculated $O_{\max }$ using equation (2). We performed sampling with replacement to obtain bootstrap standard deviation for $P_{\max }$ and $O_{\max }$ and implemented a bias-corrected and accelerated approach 13 to calculate the $95 \%$ CIs, as shown in Table 3 . The bias-corrected and accelerated approach is a method of obtaining approximate CIs using the percentiles from the bootstrap histogram. The time required for bootstrap resampling depends on the number of bootstrap iterations and sample size. Using the data from the nicotine study in rats described in this paper, one iteration took around $4.4 \mathrm{~s}$ to complete using a single-core $2.8 \mathrm{GHz}$ Intel Xeon X5560 Nehalem EP processor and 1000 iterations took around $73 \mathrm{~min}$ (4348 s).

\subsection{Bayesian hierarchical approach}

We used the same notation as described in Section 3.1. Assuming that log consumption $y_{i j}$ 's for each rat at different price $P_{j}$ are independently distributed with mean $\eta_{i j}$ and variance $\sigma^{2}$ $=1 / \tau$. In the data we used in this study, the model can be written as

$$
\eta_{i j}=\left(C_{0}+C_{M} I\left(\text { Male }_{i}\right)+u_{i}\right)+e^{\log K}\left\{e^{\left.-\left[\alpha_{0}+\alpha_{M} I\left(\mathrm{Male}_{i}\right)+b_{i}\right) P_{j}\right]}-1\right\}
$$

Let $\pi(\theta)$ be the prior joint distribution of $C_{0}, C_{M}, u_{j}, \log K, a_{0}, a_{M}, b_{i}, \tau$ where $\theta=\left(C_{0}, C_{M}\right.$, $\left.u_{i}, \log K, a_{0}, a_{M}, b_{i}, \tau\right)$. The random effects follow a multivariate normal:

$$
\left(\begin{array}{l}
\boldsymbol{u}_{\boldsymbol{i}} \\
\boldsymbol{b}_{\boldsymbol{i}}
\end{array}\right) \sim M V N\left[\left(\begin{array}{l}
0 \\
0
\end{array}\right), \Sigma\right], \Sigma^{-1} \sim \operatorname{Wishart}(\boldsymbol{\Omega}, p)
$$


where $\Omega$ is a scale matrix, a prior guess for the covariance matrix and $p$ is the degrees of freedom. The likelihood can be written as

$$
L(Y \mid \theta)=\prod_{i=1}^{N} \prod_{j=1}^{M}\left[f\left(y_{i j} \mid \eta_{i j}, \tau\right)\right]
$$

Thus, the posterior joint distribution of $C_{0}, C_{M}, u_{i}, \log K, a_{0}, a_{M}, b_{i}, \tau$ given the observations is proportional to

$$
\prod_{i=1}^{N} \prod_{j=1}^{M}\left[f\left(y_{i j} \mid \eta_{i j}, \tau\right)\right] \pi\left(C_{0}\right) \pi\left(C_{M}\right) \pi\left(u_{i}\right) \pi\left(b_{i}\right) \pi(\log K) \pi\left(a_{0}\right) \pi\left(a_{M}\right) \pi(\tau)
$$

Posterior computation was done using MCMC methods 14 and carried out using downloadable free software JAGS (http://mcmc-jags.sourceforge.net) 17 and the rjags package in $\mathrm{R}$ (http://www.r-project.org/). 18 The model code is available upon request. The burn-in consisted of 10,000 iterations; 50,000 subsequent iterations were used to compute the posterior summaries of the model parameter estimates. Convergence of MCMC was assessed using the Gelman and Rubin convergence statistic19 with three chains. The GR diagnostics for all parameter estimations are all close 1, which confirms that the chains have converged. The posterior credible intervals for $C_{0}, C_{M}, a_{0}, a_{M}$, and $\log K$ are obtained from the posterior distributions approximated by the posterior samples by using the MCMC chains.

\section{Illustration}

We assume that $y_{i j}$ is normally distributed with mean $\eta_{i j}$ and variance $\sigma^{2}=1 / \tau$. Noninformative prior distributions were specified as $\tau \sim \operatorname{gamma}\left(10^{-3}, 10^{-3}\right), C_{0} \sim \mathrm{N}\left(0,10^{3}\right), C_{M}$ $\sim \mathrm{N}\left(0,10^{3}\right), a_{0} \sim \mathrm{N}\left(0,10^{3}\right), a_{M} \sim \mathrm{N}\left(0,10^{3}\right)$, and $\log K \sim \mathrm{N}\left(0,10^{3}\right)$. The random effects for each rat $\left(\begin{array}{l}u_{i} \\ b_{i}\end{array}\right)$ were assumed to follow a bivariate normal distribution:

$$
\operatorname{MVN}\left(\left(\begin{array}{l}
0 \\
0
\end{array}\right), \Sigma\right), \Sigma^{-1} \sim \text { Wishart }\left(\left[\begin{array}{cc}
0.1 & 0.005 \\
0.005 & 0.1
\end{array}\right], 3\right)
$$

Based on the $5 \times 10^{4}$ MCMC samples, Figure 1 presents the posterior distributions for $C_{0}$, $C_{M}, a_{0}, a_{M}, \log K$, and $\tau$. The posterior distribution of $P_{\max }$ can be obtained based on equation (3) using the $5 \times 10^{4} \mathrm{MC}$ samples for $a_{0}$ and $\log K$. Likewise, $O_{\max }$ was calculated using equation (2) from the posterior samples of $P_{\max }, C_{0}, a_{0}$, and $\log K$. The values of $P_{\max }$ and $O_{\max }$ and their corresponding standard deviations for male and female rats are shown in Table 3 (right panel). The conclusions of analyzing the experimental data using Bayesian approach are consistent with the results from NLIN and the mixed effects regression.

Figure 2 presents the posterior distributions for $P_{\max }$ and $O_{\max }$ for male and female rats, respectively. These distributions could not have been obtained by using a mixed effects regression approach. Figures 3 and 4 show the demand curve for each rat on log scale, with 
95\% equal-tail credible interval for log consumption of nicotine for each rat. These figures indicate that $\log$ nicotine consumption for all the rats in the data was well described using the Bayesian approach.

In addition, we calculated $R^{2}$ as the square of the correlation between the observed and predicted $\log$ consumption. The $R^{2}$ for three methods are $0.9747,0.0 .9865$, and 0.9866 for the NLIN, mixed effects model, and the Bayesian approach, respectively, as shown in Figure 5. These results are consistent with the conclusion in Grebenstein et al.5 The results further support Hursh's claims that the demand curve offers a useful approach to model drug abuse policy in animals and humans. ${ }^{8,16}$

To further validate the models, we applied leave-one-out cross-validation to assess the predicative performance of the models. We implemented two cross-validation approaches. In the first approach, we randomly set aside one observation and used the remaining observed values to fit models for each rat. Then, we calculated the mean absolute error (MAE) of the prediction for the holdout sample. We implemented 1000 cross-validation iterations. The MAEs (standard error) are 0.254 (0.0018), 0.209 (0.0014), and 0.203 (0.0013) for NLIN, mixed effects model, and the Bayesian approach, respectively. In the second approach, we hold out a randomly selected rat at a time and implemented 1000 cross-validation iterations. In the leave-a-rat-out-at-a-time approach, the MAEs are 0.551 (0.0054), 0.478 (0.0055), and 0.485 (0.0044) for NLIN, mixed effects model, and the Bayesian approach, respectively. According to the MAEs reported from both cross-validation approaches, the mixed effects model and Bayesian approach have comparable predicative performance, and both methods outperform the NLIN approach.

\section{Simulation studies}

We generated 1000 datasets with an even number of rats, half of which were males and the remaining half were females. Nicotine consumption measured at 10 various prices for all rats. The true values for $C_{0}, C_{M}, a_{0}, a_{M}, \log K$ were set at $1,-0.3,10,-2,1.8$, respectively. Five sets of simulation studies were done by varying the sample sizes from 10 rats to 50 rats, at an increment of 10 rats each time. The random effects for each rat were generated from a bivariate normal distribution, and specifically the random effects were

$$
\left[\begin{array}{l}
\boldsymbol{u}_{\boldsymbol{i}} \\
\boldsymbol{b}_{\boldsymbol{i}}
\end{array}\right] \sim N\left(\left[\begin{array}{l}
0 \\
0
\end{array}\right],\left[\begin{array}{cc}
0.1 & 0.005 \\
0.005 & 0.1
\end{array}\right]\right)
$$

We analyzed the simulated datasets using three approaches: NLIN, mixed effects model with ML, and the Bayesian approach via the MCMC method.

The mean relative biases (MRBs) for the three approaches are shown in Table 4. The MRB is calculated as the mean difference between the true parameter and the estimated values divided by the value of the true parameter. Based on the simulation results, the NLIN approach demonstrates relatively large biases compared to the mixed effects and Bayesian hierarchical models. With increasing sample size, the biases for $\log K$ are larger using the NLIN approach. Consequently, due to the bias in estimating $\log K$, the biases for $a_{0}, a_{M}$ also 
grow large with increasing sample size. In addition, the biases for estimating the reinforcing strength of female rats $\left(a_{0}\right)$ are the largest among all parameter estimations. On the other hand, the estimations for $C_{0}, C_{M}$ are less affected by the bias in estimating $\log K$ in the NLIN approach; the MRBs for estimating $C_{0}, C_{M}$ are smaller when the sample size increases. Compared to NLIN, both mixed effects and Bayesian hierarchical models demonstrate relatively small biases (less than 4\%) in parameter estimations, and the results for the two approaches are similar.

The empirical 95\% coverage probabilities and the length of CIs or equal-tail CIs using the three approaches were calculated and are shown in Table 5. The empirical 95\% coverage probability is the percent of the $95 \% \mathrm{CI}$ or credible intervals (equal-tail CI) covering the true parameter based on 1000 Monte Carlo simulations. In the NLIN approach, $\log K$ is calculated based on the range of consumption; hence, the standard deviation and CIs cannot be obtained (shown as NA). As indicated by the simulation results, the NLIN approach demonstrates a relatively low $95 \%$ CI coverage rate compared to the other two alternative approaches. The $95 \%$ coverage rates are the lowest for $a_{0}$. Furthermore, the lengths of CIs obtained by the NLIN approach are longer than the other two alternative approaches. The simulation results shown in Table 5 suggest that the Bayesian hierarchical approach provides better $95 \%$ credible interval coverage rates for the estimation of $C_{0}, C_{M}$. On the other hand, for $a_{0}, a_{M}$ estimation, the mixed effect model reports longer CI lengths and hence achieves better empirical $95 \%$ CI coverage probability.

To compare the robustness of the three methods when model is mis-specified, we simulated data with 100 rats under the model below10

$$
\log Q_{i j}=\log l+b \log p_{j}-a p_{j}+\varepsilon_{i j}
$$

$i=1,2, \ldots 100$ and $j=1,2, \ldots 10$. In the above equation, $Q_{i j}$ is the consumption for the $t$ th rat at the $j$ th price point. The predictive consumptions were obtained using the three models. The MAEs of predictive values are 22.128, 11.410, and 11.425 for NLIN, mixed effects model, and the Bayesian approach, respectively. The mixed effects model and Bayesian approach are much more robust than the NLIN approach when the model is mis-specified. In summary, our simulation analyses suggest that the Bayesian model and the mixed-effect model have comparable performance and both achieve better performance than NLIN approach.

\section{Discussion}

In this paper, we compared three existing approaches for analyzing demand curve data. In the analysis results from animal NSA data analysis, both mixed effects and Bayesian hierarchical approach are comparable. In the mixed effects regression framework, a general form of the exponential demand function can be expressed as:

$$
y_{i j}=\left(C_{0}+\sum_{k=1}^{p} \beta_{k} X_{k}+u_{i}\right)+e^{\log K}\left[e^{-\left(\alpha_{0}+\sum_{k=1}^{q} \gamma_{k} Z_{k}+b_{i}\right) P_{j}}-1\right]+\varepsilon_{i j}
$$


In this equation, $C_{0}$ is the original $\log$ consumption with price at zero; $X_{k}$ and $Z_{k}$ are the covariates that contribute to the differences of log consumption and rate of decline, respectively; $\beta_{k}$ and $\gamma_{k}$ are the corresponding coefficients. For the Bayesian approach, a general form for estimating the exponential demand curve can be expressed as

$$
\eta_{i j}=\left(C_{0}+\sum_{k=1}^{p} \beta_{k} X_{k}+u_{i}\right)+e^{\log K}\left[e^{-\left(\alpha_{0}+\sum_{k=1}^{q} \gamma_{k} Z_{k}+b_{i}\right) P_{j}}-1\right] .
$$

The Bayesian approach provides direct statistical inference for two clinical relevant quantities: $P_{\max }$ and $O_{\max }$ through sampling from posterior distributions. Additional biascorrected and accelerated bootstrap analysis is required to obtain 95\% confidence for $P_{\max }$ and $O_{\max }$ through a mixed effects regression model. The predictive performance using crossvalidation indicated that both Bayesian and mixed effects methods achieve smaller MAEs than the NLIN approach.

The computational time required for implementing NLIN, mixed effects, and Bayesian model using the data described in this paper are $0.21 \mathrm{~s}, 4362 \mathrm{~s}$, and $3877 \mathrm{~s}$ in a single-core $2.8 \mathrm{GHz}$ Intel Xeon X5560 Nehalem EP processor. Although the computational time for the mixed effect model and Bayesian approach are longer than the conventional NLIN approach, the simulation analyses (Tables 4 and 5) indicate that with small-to-moderate sample sizes, the popularly used NLIN approach demonstrates relatively large estimation biases and a low 95\% CI coverage rate. The performances of mixed effects regression and Bayesian hierarchical approaches are comparable. The Bayesian approach provides posterior distributions for all estimated parameters. In addition, the advantages of using the Bayesian approach are more pronounced, when the sample size is small. With current advances in computing capacities and modern computational algorithms via MCMC, the Bayesian method can be easily implemented, and thus should be considered a good alternative when estimating the demand curve in public health research.

\section{Acknowledgement}

The authors thank Dr. Mark LeSage for providing raw data from the nicotine self-administration study in rats for demand curve analysis, and for his comments on early versions of the manuscript.

Funding

The author(s) disclosed receipt of the following financial support for the research, authorship, and/or publication of this article: The authors would like to acknowledge the funding support of NIH 1U54-DA031659-01 and U19CA157345 for this work. Research reported in this publication was also supported by NIH grant P30 CA77598 utilizing the Biostatistics and Bioinformatics Core shared resource of the Masonic Cancer Center, University of Minnesota, and by the National Center for Advancing Translational Sciences of the National Institutes of Health Award Number UL1TR000114.

\section{References}

1. Griffiths RR, Brady JV and Bradford LD. Predicting the abuse liability of drugs with animal drug self-administration procedures: Psychomotor stimulants and hallucinogens In: Thompson T and Dews PB (eds) Advances in behavioral pharmacology. Vol. 2, New York: Academic Press, 1979, pp. 163-208. 
2. Katz JL. Models of relative reinforcing efficacy of drugs and their predictive utility. Behav Pharmacol 1990; 1: 283-301. [PubMed: 11175414]

3. Bentzley BS, Jhou TC and Aston-Jones G. Economic demand predicts addiction-like behavior and therapeutic efficacy of oxytocin in the rat. PNAS 2014; 111: 11822-11827. [PubMed: 25071176]

4. Hursh SR, Raslear TG, Shurtleff D, et al. A cost-benefit analysis of demand for food. J Exp Anal Behav 1988; 50: 419-440. [PubMed: 3209958]

5. Grebenstein P, Burroughs D, Zhang Y, et al. Sex differences in nicotine self-administration in rats during progressive unit dose reduction: implications for nicotine regulation policy. Pharmacol Biochem Behav 2013; 114: 70-81. [PubMed: 24201048]

6. Donny EC, Taylor TG, LeSage MG, et al. Impact of tobacco regulation on animal research: new perspectives and opportunities. Nicotine Tob Res 2012; 14: 1319-1338. [PubMed: 22949581]

7. Murphy JG, MacKillop J, Skidmore JR, et al. Reliability and validity of a demand curve measure of alcohol reinforcement. Exp Clin Psychopharmacol 2009; 17: 396-404. [PubMed: 19968404]

8. Hursh SR and Roma PG. Behavioral economics and empirical public policy. J Exp Anal Behav 2013; 99: 98-124. [PubMed: 23344991]

9. Hursh SR and Silberberg A. Economic demand and essential value. Psychol Rev 2008; 115: 186198. [PubMed: 18211190]

10. Yu J, Liu L, Collins RL, et al. Analytical problems and suggestions in the analysis of behavioral economic demand curves. Multivar Behav Res 2014; 49: 178-192.

11. Bentzley BS, Fender KM and Aston-Jones G. The behavioral economics of drug selfadministration: a review and new analytical approach for within-session procedures. Psychopharmacology (Berl) 2013; 226: 113-125. [PubMed: 23086021]

12. Liao W, Luo X, Le CT, et al. Analysis of cigarette purchase task instrument data with a leftcensored. Exp Clin Psychopharmacol 2013; 21: 124-132. [PubMed: 23356731]

13. DiCiccio TJ and Efron B. Bootstrap confidence intervals (with discussion). Stat Sci 1996; 11: 189228.

14. Gelfand AE and Smith AFM. Sampling-based approaches to calculating marginal densities. JASA 1990; 85: 398-409.

15. Ashby D Bayesian statistics in medicine: a 25 year review. Stat Med 2006; 25: 3589-3631. [PubMed: 16947924]

16. Hursh SR. Behavioral economics of self-drug-administration and drug abuse policy. J Exp Anal Behav 1991; 56: 377-393. [PubMed: 1955823]

17. Plummer M. JAGS: a program for analysis of Bayesian graphical models using Gibbs sampling; Proceedings of the 3rd international workshop on distributed statistical computing; Vienna, Austria. 20-22 March 2003;

18. Lunn D, Spiegelhalter D, Thomas A, et al. The BUGS project: evolution, critique and future directions. Stat Med 2009; 28: 3049-3067. [PubMed: 19630097]

19. Gelman A and Rubin DB. Inference from iterative simulation using multiple sequences. Stat Sci 1992; 7: 457-472. 

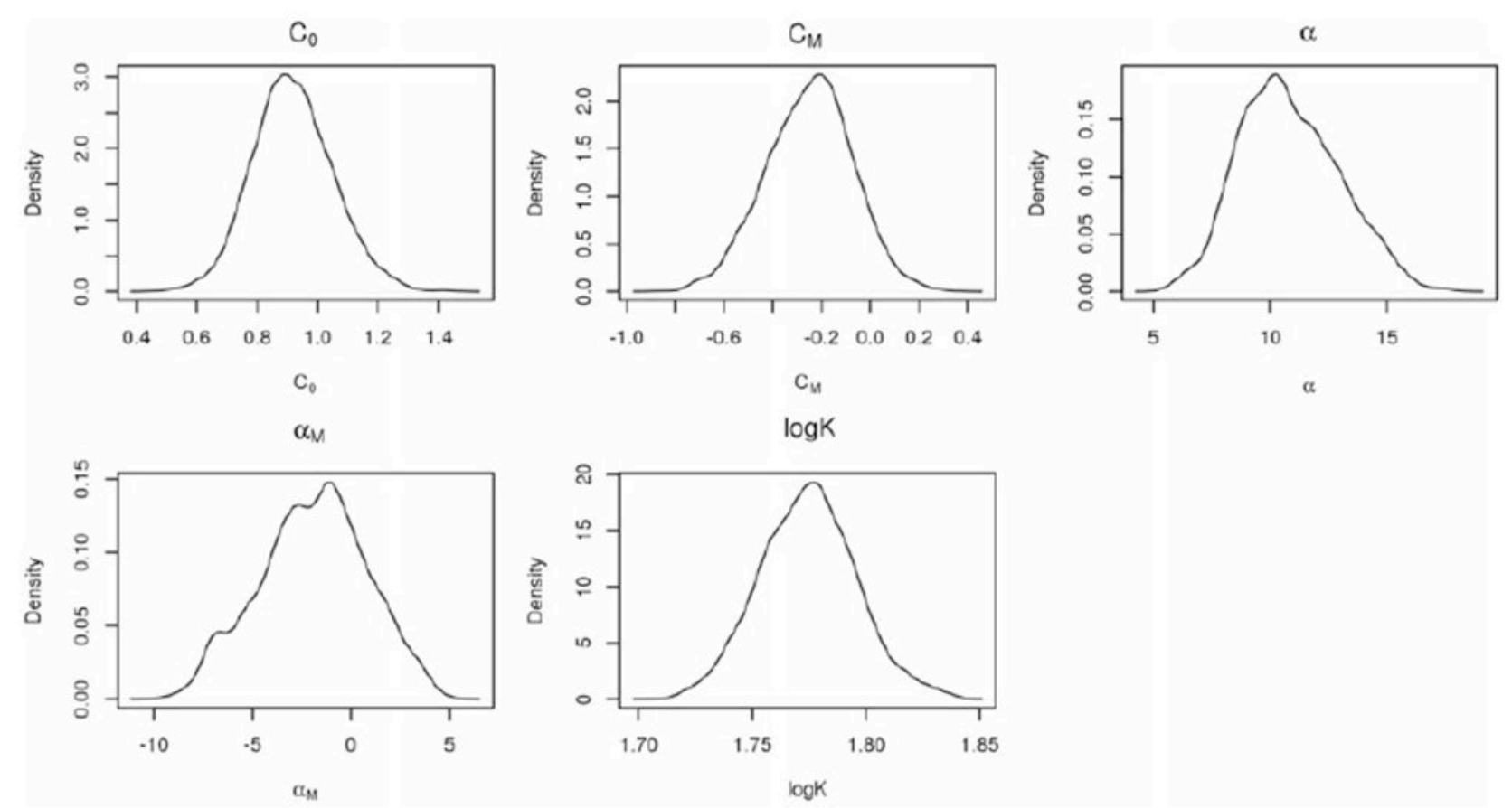

Figure 1.

Posterior distributions for demand curve parameter estimates. 

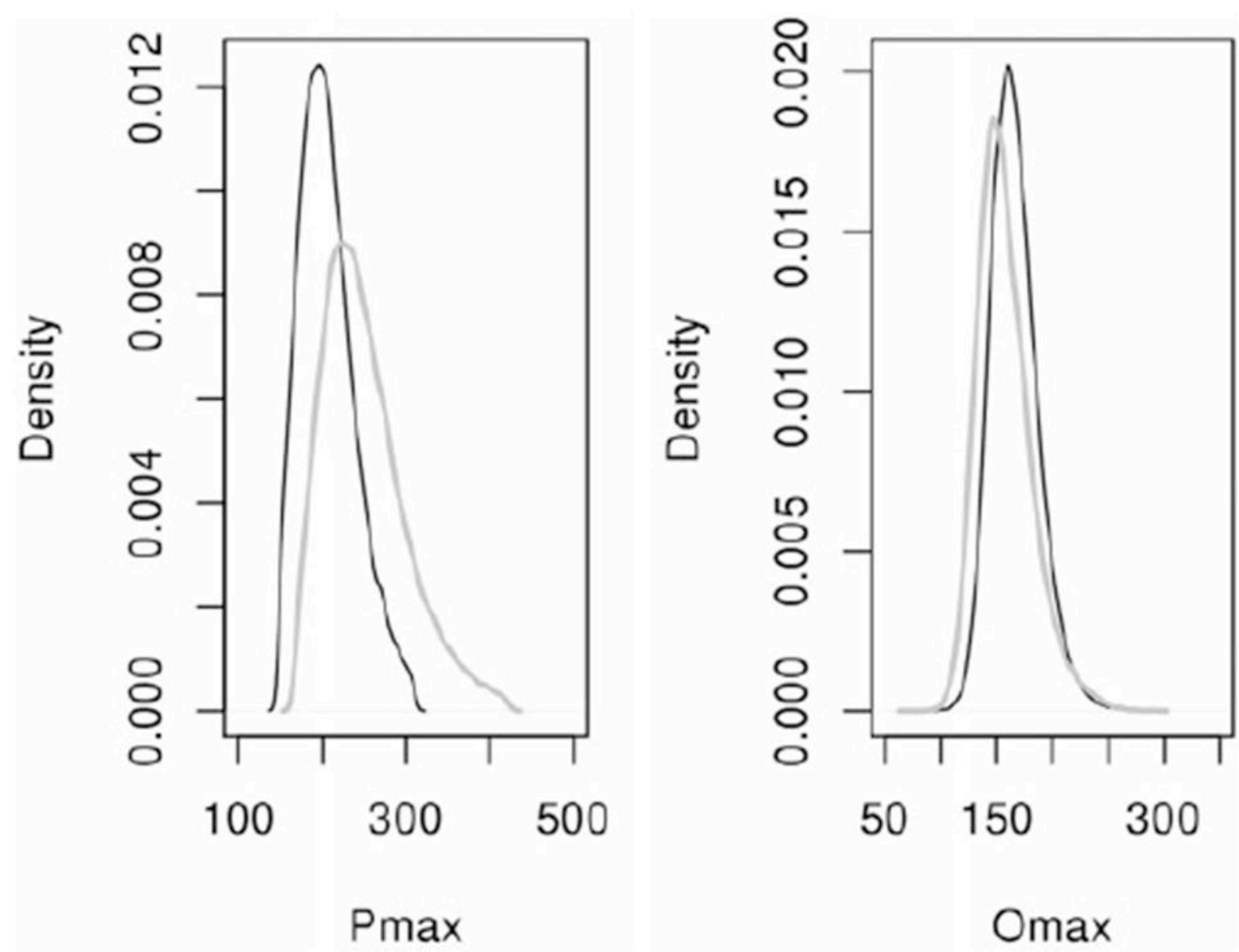

Figure 2.

Posterior distributions of $P_{\max }$ and $O_{\max }$ for male (gray line) and female (black line) rats. 


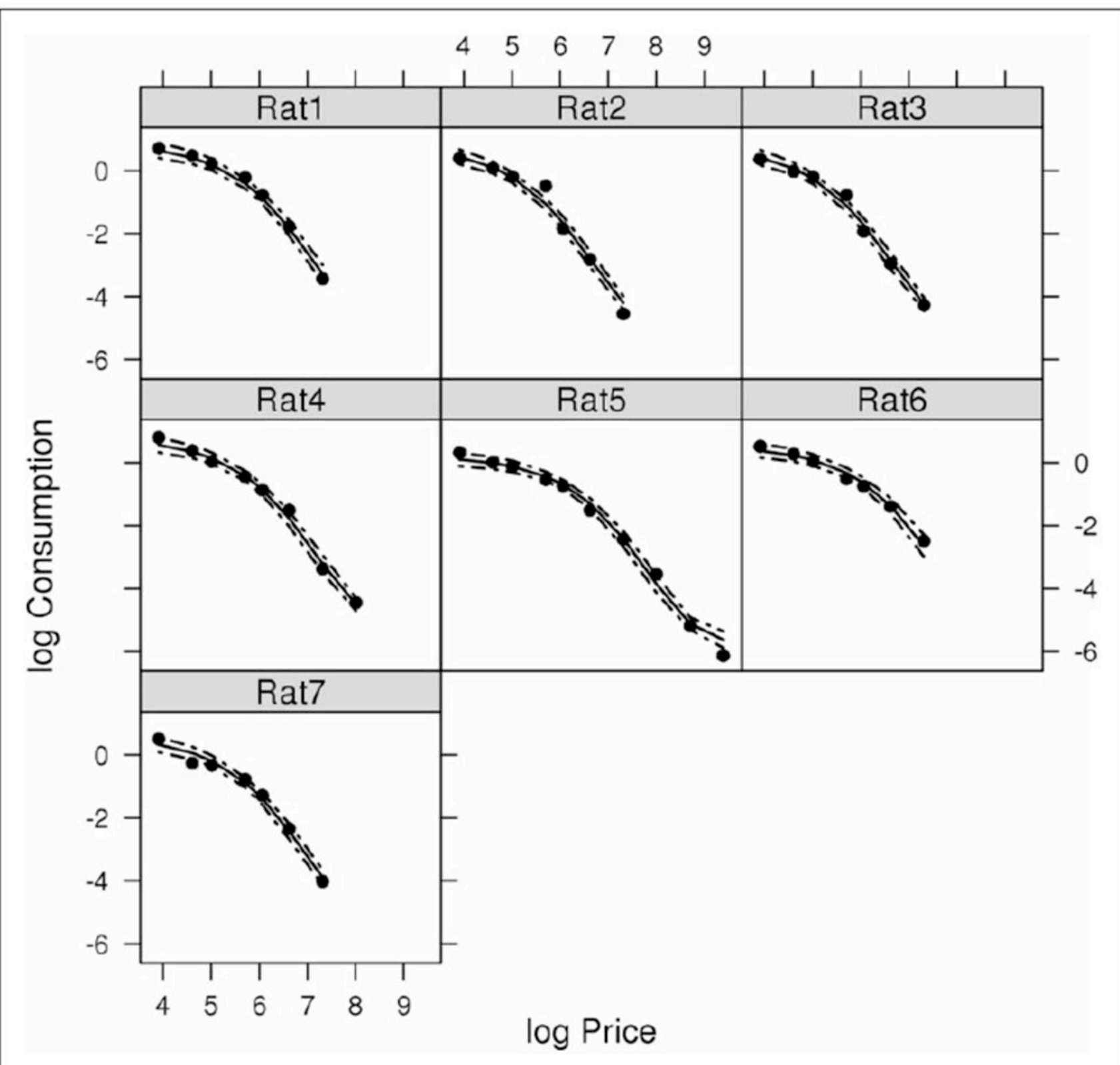

Figure 3.

Demand curve for each male rat with $95 \%$ credible interval. Black dots represent observed value; solid lines are the predicted values from the Bayesian model with $95 \%$ credible intervals indicated by dotted line. 


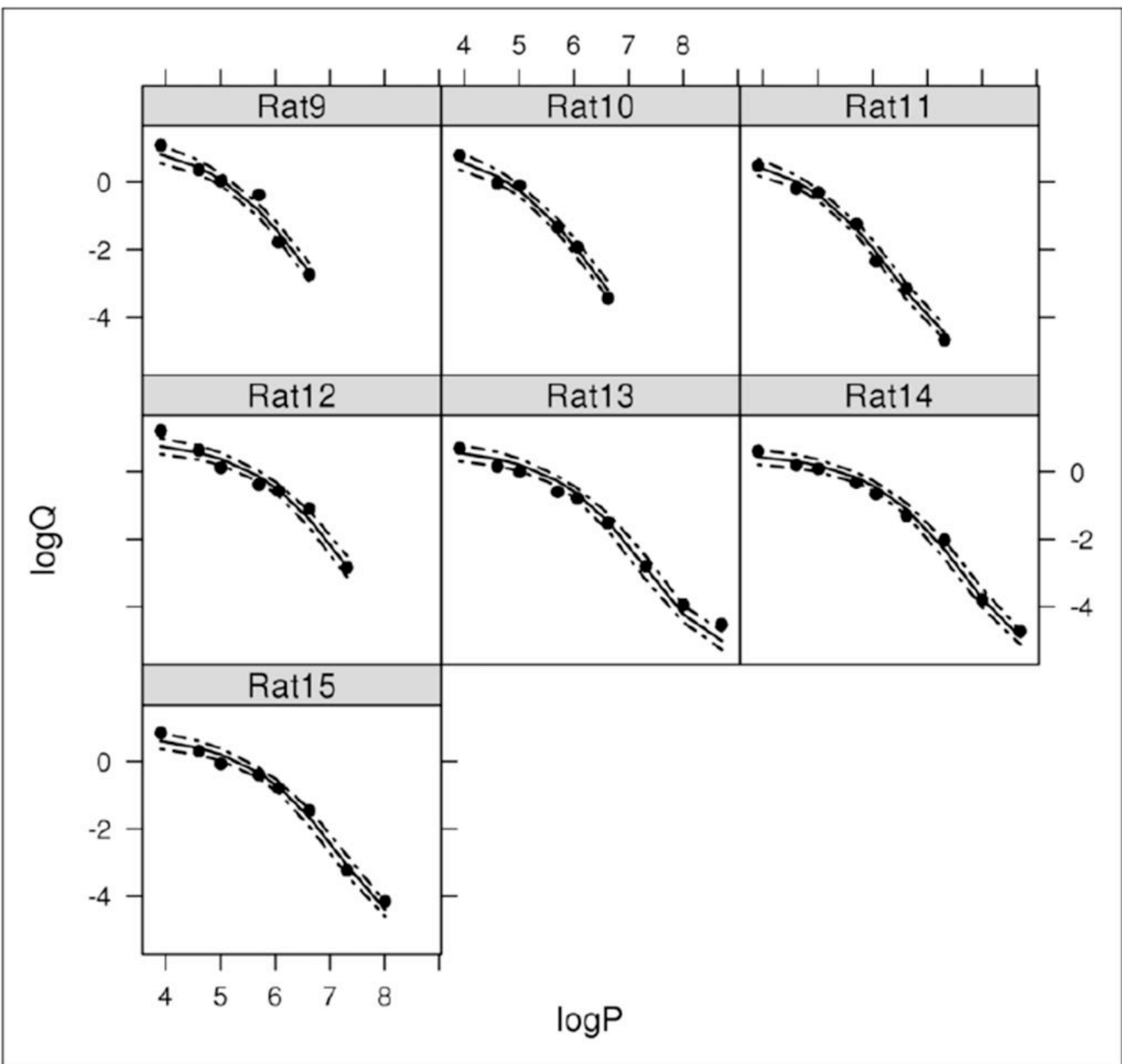

Figure 4.

Demand curve for each female rat with $95 \%$ credible interval. Black dots represent observed value; solid lines are the predicted values from the Bayesian model with $95 \%$ credible intervals indicated by dotted line. 


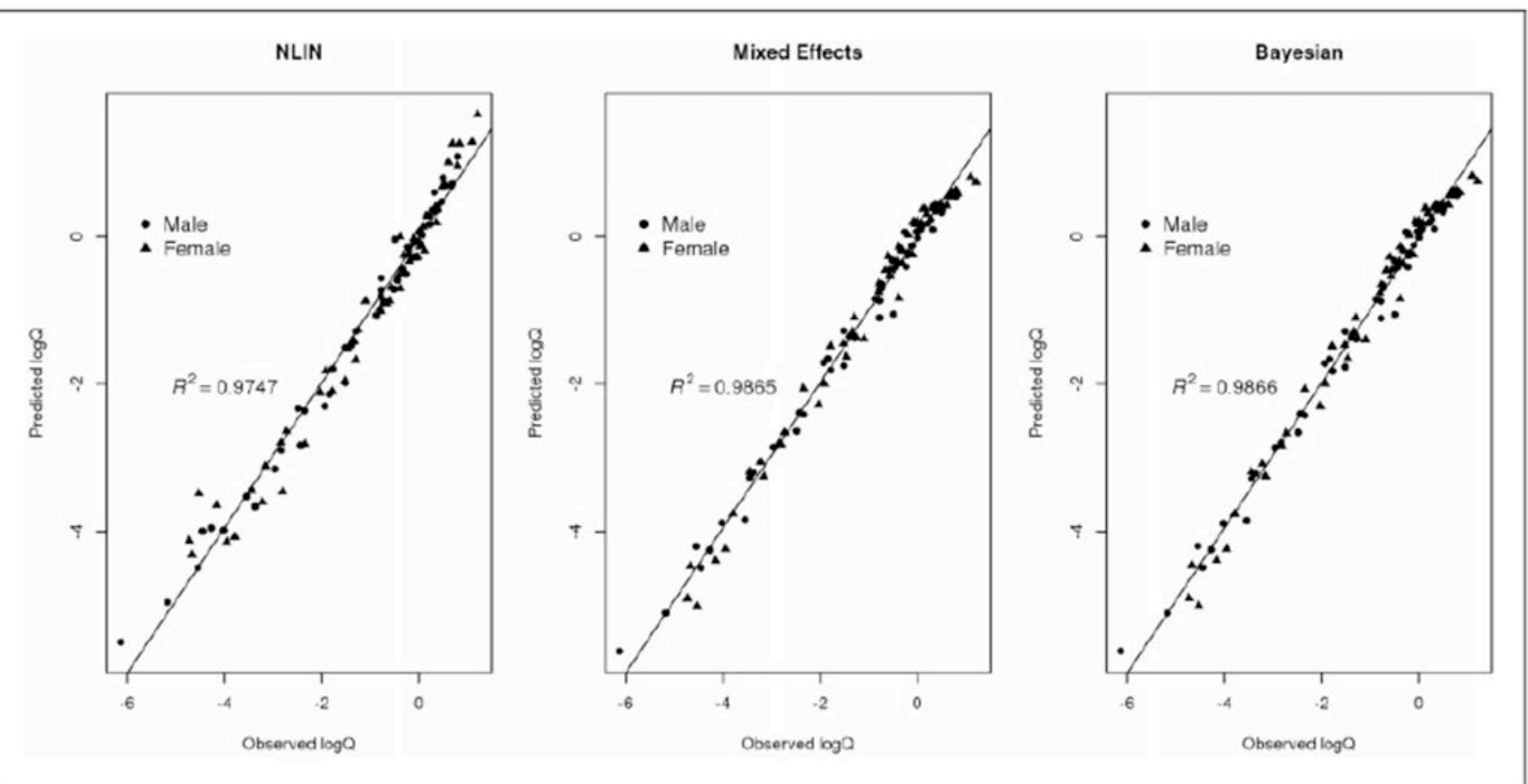

Figure 5.

Predicted log nicotine consumed vs. observed values for NLIN, mixed effects model, and Bayesian approach. 


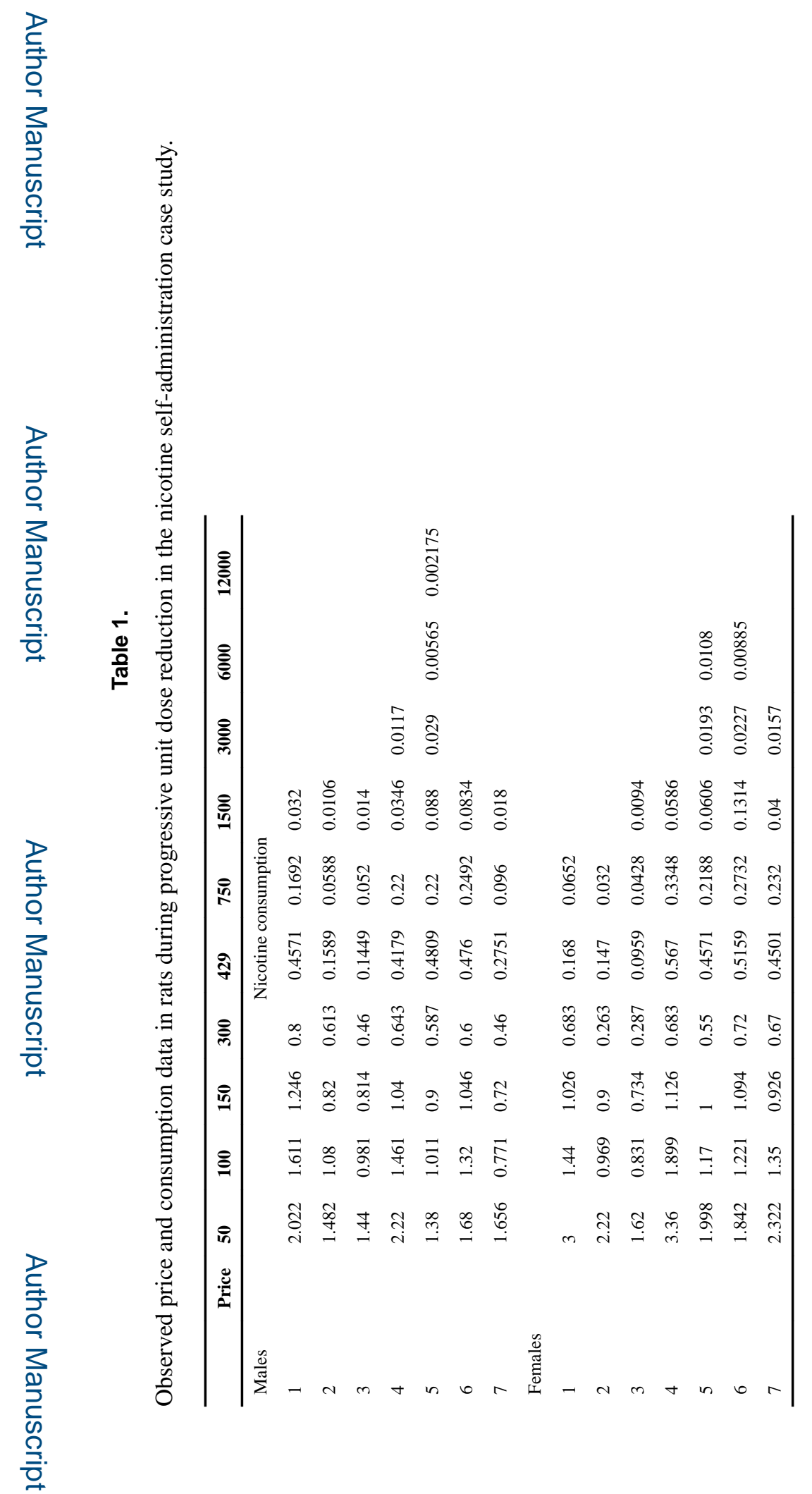

Stat Methods Med Res. Author manuscript; available in PMC 2018 November 28. 
Table 2.

Demand curve parameter estimates and their corresponding standard errors and 95\% CI/equal-tail CI for rats during progressive unit dose reduction in the nicotine self-administration case study.

\begin{tabular}{|c|c|c|c|c|c|c|c|}
\hline \multirow[b]{3}{*}{ Parameter } & \multicolumn{7}{|l|}{ Method } \\
\hline & \multicolumn{2}{|c|}{ Non-linear least square regression } & \multicolumn{2}{|c|}{ Mixed effects regression } & \multicolumn{3}{|c|}{ Bayesian hierarchical approach } \\
\hline & Estimate $(95 \%$ CI $)$ & SE & Estimate (95\% CI) & SE & DF & Estimate (95\% equal-tail CI) & SE \\
\hline$C_{0}$ & $2.69(1.81,3.56)$ & 0.36 & $0.91(0.66,1.16)$ & 0.12 & 12 & $0.92(0.69,1.16)$ & 0.12 \\
\hline$C_{M}$ & $0.67(-0.25,1.59)$ & 0.41 & $-0.25(-0.60,0.10)$ & 0.16 & 12 & $-0.28(-0.59,0.04)$ & 0.16 \\
\hline$a_{0}$ & $4.40(2.63,6.17)$ & 0.72 & $10.54(6.58,14.50)$ & 1.83 & 12 & $10.70(7.19,13.92)$ & 1.71 \\
\hline$a_{M}$ & $-0.03(-2.00,1.94)$ & 0.89 & $-1.93(-7.33,3.48)$ & 2.48 & 12 & $-2.46(-6.47,1.83)$ & 2.11 \\
\hline $\log K$ & 2.50 & NA & $1.77(1.73,1.82)$ & 0.02 & 12 & $1.77(1.73,1.82)$ & 0.02 \\
\hline
\end{tabular}

Note: The estimates and SEs are multiplied by $10^{4}$ in all methods. 
Table 3.

$P_{\max }$ and $O_{\max }$ estimates and their corresponding standard deviations for the case study.

\begin{tabular}{|c|c|c|c|}
\hline \multirow[b]{2}{*}{ Parameter } & \multicolumn{3}{|l|}{ Method } \\
\hline & $\begin{array}{l}\text { Non-linear least square regression } \\
\text { Estimate }(95 \% \mathrm{CI})\end{array}$ & $\begin{array}{l}\text { Mixed effects regression Estimate } \\
(95 \% \text { CI })\end{array}$ & $\begin{array}{l}\text { Bayesian hierarchical approach } \\
\text { Estimate (95\% equal-tail CI) }\end{array}$ \\
\hline$P_{\max }$ for Male rats & $293.23(151.08,435.38)$ & $243.13(220.00,364.45)$ & $255.18(241.72,422.31)$ \\
\hline$P_{\max }$ for Female rats & $240.34(116.28,364.40)$ & $198.70(152.09,292.60)$ & $209.07(148.02,311.23)$ \\
\hline$O_{\max }$ for Male rats & $172.94(126.36,219.52)$ & $153.73(125.72,267.47)$ & $159.64(116.74,236.37)$ \\
\hline$O_{\max }$ for Female rats & $183.11(124.50,241.73)$ & $161.66(117.80,216.70)$ & $167.29(129.08,225.16)$ \\
\hline
\end{tabular}


Table 4.

Mean relative errors for demand curve parameters based on 1000 datasets.

\begin{tabular}{|c|c|c|c|c|}
\hline Number of rats in each simulation & Parameter & Non-linear least square & Mixed Effects & Bayesian approach \\
\hline \multirow[t]{5}{*}{$N=10$} & $a_{0}$ & -0.27 & 0.0061 & 0.0106 \\
\hline & $a_{M}$ & 0.06 & 0.0086 & 0.0140 \\
\hline & $\log K$ & 0.12 & 0.0003 & -0.0002 \\
\hline & $C_{0}$ & 0.18 & 0.0006 & 0.0057 \\
\hline & $C_{M}$ & 0.59 & -0.0126 & 0.0000 \\
\hline \multirow[t]{5}{*}{$N=20$} & $a_{0}$ & -0.35 & -0.0017 & 0.0009 \\
\hline & $a_{M}$ & -0.05 & -0.0240 & -0.0195 \\
\hline & $\log K$ & 0.14 & 0.0003 & 0.0000 \\
\hline & $C_{0}$ & 0.11 & -0.0034 & -0.0021 \\
\hline & $C_{M}$ & 0.57 & -0.0280 & -0.0303 \\
\hline \multirow[t]{5}{*}{$N=30$} & $a_{0}$ & -0.40 & 0.0017 & 0.0029 \\
\hline & $a_{M}$ & -0.10 & 0.0109 & 0.0125 \\
\hline & $\log K$ & 0.15 & -0.0001 & -0.0003 \\
\hline & $C_{0}$ & 0.06 & 0.0087 & 0.0046 \\
\hline & $C_{M}$ & 0.63 & 0.0385 & 0.0215 \\
\hline \multirow[t]{5}{*}{$N=40$} & $a_{0}$ & -0.43 & 0.0020 & 0.0030 \\
\hline & $a_{M}$ & -0.16 & -0.0006 & 0.0005 \\
\hline & $\log K$ & 0.16 & 0.0002 & 0.0000 \\
\hline & $C_{0}$ & 0.03 & 0.0039 & 0.0055 \\
\hline & $C_{M}$ & 0.55 & -0.0053 & -0.0041 \\
\hline \multirow[t]{4}{*}{$N=50$} & $a_{0}$ & -0.46 & 0.0005 & 0.0016 \\
\hline & $a_{M}$ & -0.21 & 0.0010 & 0.0015 \\
\hline & $\log K$ & 0.17 & 0.0003 & 0.0002 \\
\hline & $C_{0}$ & -0.01 & 0.0035 & 0.0044 \\
\hline
\end{tabular}


Table 5.

Empirical coverage probability of 95\% confidence/credible interval and interval lengths based on 1000 datasets.

\begin{tabular}{|c|c|c|c|c|c|c|c|}
\hline \multirow{2}{*}{$\begin{array}{l}\text { Number } \\
\text { of rats in } \\
\text { each } \\
\text { simulation }\end{array}$} & \multirow[b]{2}{*}{ Parameter } & \multicolumn{2}{|l|}{$\underline{\text { nonlinear least square }}$} & \multicolumn{2}{|l|}{ Mixed effects } & \multicolumn{2}{|l|}{ Bayesian approach } \\
\hline & & Coverage probability & CI length & Coverage probability & CI length & Coverage probability & Equal-tail CI length \\
\hline \multirow[t]{5}{*}{$N=10$} & $a_{0}$ & 0.18 & 3.05 & 0.97 & 2.95 & 0.94 & 2.55 \\
\hline & $a_{M}$ & 0.89 & 3.75 & 0.97 & 3.78 & 0.95 & 3.27 \\
\hline & $C_{0}$ & 0.74 & 0.76 & 0.88 & 0.57 & 0.93 & 0.66 \\
\hline & $C_{\mathrm{M}}$ & 0.86 & 1.07 & 0.88 & 0.78 & 0.94 & 0.92 \\
\hline & $\log K$ & NA & NA & 0.98 & 0.07 & 0.95 & 0.06 \\
\hline \multirow[t]{4}{*}{$N=20$} & $a_{0}$ & 0.01 & 2.29 & 0.96 & 1.90 & 0.95 & 1.77 \\
\hline & $a_{M}$ & 0.89 & 2.71 & 0.96 & 2.43 & 0.95 & 2.26 \\
\hline & $C_{M}$ & 0.85 & 0.83 & 0.89 & 0.57 & 0.95 & 0.63 \\
\hline & $\log K$ & NA & NA & 0.96 & 0.05 & 0.95 & 0.04 \\
\hline \multirow[t]{5}{*}{$N=30$} & $a_{0}$ & 0.00 & 1.93 & 0.96 & 1.51 & 0.95 & 1.44 \\
\hline & $a_{M}$ & 0.84 & 2.20 & 0.96 & 1.93 & 0.95 & 1.83 \\
\hline & $C_{0}$ & 0.77 & 0.51 & 0.87 & 0.34 & 0.94 & 0.37 \\
\hline & $C_{M}$ & 0.81 & 0.73 & 0.88 & 0.47 & 0.94 & 0.52 \\
\hline & $\log K$ & NA & NA & 0.95 & 0.04 & 0.94 & 0.04 \\
\hline \multirow[t]{4}{*}{$N=40$} & $a_{0}$ & 0.00 & 1.66 & 0.94 & 1.29 & 0.94 & 1.24 \\
\hline & $a_{M}$ & 0.78 & 1.88 & 0.96 & 1.65 & 0.95 & 1.58 \\
\hline & $C_{M}$ & 0.82 & 0.64 & 0.89 & 0.41 & 0.94 & 0.45 \\
\hline & $\log K$ & NA & NA & 0.95 & 0.03 & 0.95 & 0.03 \\
\hline \multirow[t]{5}{*}{$N=50$} & $a_{0}$ & 0.00 & 1.47 & 0.95 & 1.14 & 0.94 & 1.10 \\
\hline & $a_{M}$ & 0.72 & 1.64 & 0.96 & 1.45 & 0.95 & 1.41 \\
\hline & $C_{0}$ & 0.73 & 0.41 & 0.87 & 0.27 & 0.95 & 0.29 \\
\hline & $C_{M}$ & 0.82 & 0.58 & 0.90 & 0.38 & 0.95 & 0.40 \\
\hline & $\log K$ & NA & NA & 0.95 & 0.03 & 0.96 & 0.03 \\
\hline
\end{tabular}

